\title{
Comparing Automatically Extracted Topics from Online Suicidal Ideation and the Responses they Invoke
}

\author{
Elise Glaser \\ Colorado College \\ Colorado Springs, $\mathrm{CO}$ \\ e_glaser@coloradocollege.edu \\ Jonathan Gemmell \\ Depaul University \\ Chicago, IL \\ jgemmell@cdm.depaul.edu
}

\author{
Alexander Morain \\ University of Illinois \\ Champaign, IL \\ amorain2@illinois.edu \\ Daniela Raicu \\ Depaul University \\ Chicago, IL \\ dstan@cdm.depaul.edu
}

\begin{abstract}
Suicide is a national public health concern, claiming over one million lives each year worldwide. The ability to understand, identify, and respond to suicidal behavior remains a key priority in preventing suicide. As online social networks have grown in accessibility and popularity, it is increasingly common for users to both discuss mental health and receive support from others online. These online conversations have previously been evaluated by analyzing the language features of social media posts and detecting risk factors and levels of distress among users. In this work, we use natural language processing tools to automatically extract informal topics within posts discussing suicidal ideation and the responses to these posts. Our evaluation demonstrates that frequent topics within the posts represent psychiatrically defined risk factors for suicide, and frequent topics within the responses represent CDC recommended responses to suicidal ideation based on identified protective factors.
\end{abstract}

\section{CCS CONCEPTS}

-Information systems $\rightarrow$ Social networks; • Computing methodologies $\rightarrow$ Information extraction; • Applied computing $\rightarrow$ Psychology.

\section{KEYWORDS}

Social Networking, Suicidal Ideation, Natural Language Processing

\section{ACM Reference Format:}

Elise Glaser, Alexander Morain, Jonathan Gemmell, and Daniela Raicu. 2020. Comparing Automatically Extracted Topics from Online Suicidal Ideation and the Responses they Invoke. In The 35th ACM/SIGAPP Symposium on Applied Computing (SAC '20), March 30-April 3, 2020, Brno, Czech Republic. ACM, New York, NY, USA, 8 pages. https://doi.org/10.1145/3341105.3373902

Permission to make digital or hard copies of all or part of this work for personal or classroom use is granted without fee provided that copies are not made or distributed for profit or commercial advantage and that copies bear this notice and the full citation on the first page. Copyrights for components of this work owned by others than ACM must be honored. Abstracting with credit is permitted. To copy otherwise, or republish, to post on servers or to redistribute to lists, requires prior specific permission and/or a fee. Request permissions from permissions@acm.org.

SAC '20, March 30-April 3, 2020, Brno, Czech Republic

(c) 2020 Association for Computing Machinery.

ACM ISBN 978-1-4503-6866-7/20/03.

https://doi.org/10.1145/3341105.3373902

\section{INTRODUCTION}

Suicide is defined as "a fatal self-injurious act with some evidence of intent to die", and is the tenth leading cause of death in the United States [30]. There is a proportionally larger population of those who suffer from non-fatal suicidal behaviors. In 2017, 47,173 Americans died by suicide, while over 1.4 million Americans attempted suicide [3]. Nonfatal suicidal behaviors are classified into three categories: suicide ideation, which refers to thoughts of engaging in behavior intended to end one's life [30]; suicide plan, which refers to the formulation of a specific method through which one intends to die; and suicide attempt, which refers to engagement in potentially self-injurious behavior in which there is at least some intent to die [29].

Active suicidal ideation includes the act of identifying a method, having a plan, or having intent to act. Suicidal ideation is the most prevalent suicidal behavior with a worldwide lifetime prevalence of 9.2 percent, whereas suicide attempts have a prevalence of 2.7 percent [30]. Roughly a third of adolescents suffering from suicidal ideation go on to attempt suicide within one year.

Online social networks offer a candid look into the nature of peoples' thoughts and feelings through public posts and messages. Currently, there are over 3.5 billion social media users worldwide, most of which visit popular social networks such as Facebook, Instagram, Youtube, Twitter, Reddit, and LinkedIn [21]. Each of these websites offer a modality through which users can post public content, making social networks a robust data source for the analysis of language and topics on a variety of issues.

As online social networks have grown in accessibility and popularity, adolescents and young adults in particular have adopted these networks as modes of self-expression. Research shows that 42 percent of internet users age 18-24 use Reddit, expressing among other things self-reported mental health-related experiences, thoughts, and perspectives [14]. Furthermore, the division of the website into subreddits lends itself to specific and moderated forums.

In previous work, researchers extracted informal latent recurring topics of suicidal ideation found in posts from $\mathrm{r} /$ SuicideWatch. The prior analysis of latent topics extracted from social media surrounding suicidal ideation suggests that users in these forums express ideas that are complementary to the risk factors defined by experts, but differ in their scope and language [19]. 
In this paper, we analyze the topics drawn from language surrounding suicidal ideation and provide a comparative analysis to the responses they invoke. We seek to further characterize posts describing suicidal ideation and their responses to better understand how users respond to online suicidal ideation.

Our rigorous evaluation of posts and responses from an online suicide forum shows that distinct topics occur within posts and their respective responses. There exist stark differences between the topics extracted from suicidal posts and the topics extracted from their responses, particularly in sentiment. Finally, these topics can be mapped to risk factors and preventative methods for suicide in order to specify the nature of responses to different circumstances and sentiments within posts.

\section{RELATED WORK}

According to the CDC, suicide was the tenth leading cause of death in 2017, and the second leading cause of death for individuals between the ages of 10 to 34 [3]. The severity of this disease is compounded by the fact that for each suicide in the U.S, there are an estimated 25 suicide attempts, and 4.3 percent of adults over the age of 18 reported serious thoughts of suicide in that year.

Risk factors are defined as characteristics associated with suicide, though they are not necessarily direct causes. Predisposing factors include a family history of suicide and childhood abuse. Furthermore, a history of mental illness and/or substance abuse also increase the risk of suicide. Factors also include personal events such as financial troubles, interpersonal conflict, or physical illness [30]. In addition, feelings such as helplessness, hopelessness, isolation and worthlessness are considered key indicators of suicide. Lack of mental health treatment, due to unwillingness or inaccessibility, is considered a risk factor as well. More obvious factors include access to lethal methods and previous suicide attempts [6].

Protective factors are those that buffer individuals from suicidal thoughts and behaviors. Protective factors have not been studied to the extent of risk factors, but are considered equally as important [6]. These factors include effective clinical care for mental, physical, and substance abuse disorders and access to clinical interventions and support mechanisms. Furthermore, relationships with mental health professionals and doctors should be ongoing. Another key factor is a sense of connectedness, developed through family, community support, or social institutions. Factors also include feelings of self-esteem and a sense of purpose, as well as skills such as problem-solving skills, coping mechanisms, and the ability to adapt to change [1].

Natural language processing explores how computers may comprehend or manipulate natural language text and speech [13]. In text analysis, the goal of natural language processing is to extract both syntactic and semantic meaning from text. For example, the word embedding method used in this paper, called Word2Vec, learns words through a one-dimensional neural network based on the context of neighboring words, and represents them as distributed vectors [31]. Other software tools are used to analyze sentiment, such as LIWC (Linguistic Inquiry and Word Count), which counts words based on psychologically-grounded lists of positive and negative emotional terms [11]. Research has leveraged tools such as Word2Vec and LIWC to analyze text surrounding mental health.
Studies have taken advantage of the self-reporting nature of social media to analyze language surrounding mental health. A 2015 study obtained twitter messages with self-reported diagnoses and analyzed the text using LIWC and character n-gram language models in order to obtain quantifiable differences in the language describing different disorders [16]. Another 2017 study used Word2Vec to analyze a dataset of tweets in order to detect and classify hate speech, providing grounds for social networks to more easily detect messages with hateful sentiments [18]. These studies show that natural language processing is a powerful tool for identifying specific language as well as the sentiment expressed in online language.

A 2016 study used Reddit posts from numerous mental health subreddits, and analyzed text based off the categories of linguistic structure, interpersonal awareness, and interaction by measuring certain key words and phrases. The study was able to derive markers of shifts in the language of users who discuss general mental health concerns that later discuss suicidal ideation [17]. This research shows that online language can be used to indicate the severity of mental health issues. An earlier 2014 study sought to detect suicidal risk factors in social media posts by training text-based models on twitter posts. By representing posts with feature vectors, and extracting topics with latent Dirichlet allocation, the study was able to accurately label posts based on predefined levels of distress [20]. The findings from both these studies show the value of using social media posts as a dataset as well as the ways in which natural language processing tools can both detect and predict significant information regarding the language of mental health.

Association rule mining finds items that occur frequently together and determines the strength of their correlation [28]. Association rules have been applied to semantic web data in order to find meaningful relationships. A study from 2011 mined available web ontology language annotations on patient follow-ups for association rules, and labelled items with their ontology for deep analysis. The study found meaningful rules based on different contexts such as "patient", "report" or "visit" using labelled data and features gleaned from semantic web data [27].

\section{METHODOLOGY}

In this section we detail our approach to develop meaningful insights on the language of posts describing suicidal ideation and the language of responses to these posts by using data gained from an online support forum. We first describe our approach to embedding words as vectors, then discuss our method of topic clustering, our use of matrix multiplication to detect these topics in each post and its respective response, and our methods of association rule mining in order to find meaningful motifs to analyze.

\subsection{Word Embeddings}

Word embedding is a method used to capture both syntactic and semantic meaning of words. Methods for training word embeddings are based on the perspective that words which occur in similar contexts tend to have similar meanings [22]. Embedding typically works by representing words as vectors in an $\mathrm{n}$-dimensional space.

Word2Vec is a popular word embedding algorithm due to its ability to capture both semantic and syntactic information. We chose this algorithm because it is extremely effective in capturing 
semantic relatedness, which allows us to find latent connections and relations within our large dataset. This algorithm uses a neural network with one hidden layer to create word vectors based off surrounding words [25]. There are two approaches to training the network: the Continuous Bag of Words model uses the surrounding words to predict the target word, and the Skip-Gram model uses the target word to predict its surrounding words within a given window size.

When an input is fed to the neural network, the output nodes are evaluated using a softmax function to obtain the probability distribution of the rest of the words in the corpus. This function can be time-consuming when operating on large corpora. To address this problem, several key methods were used to offer increased efficiency [26]. We increased the function's speed by implementing Mikolov's hierarchical softmax, which uses an efficient binary tree representation of the output layer to represent each word as a leaf and their relative probabilities as nodes [26]. Negative sampling was also implemented. Negative sampling is a technique that involves only updating a certain number of output nodes for each run.

\subsection{Topic Clustering}

Topic clustering is used to extract groupings of words that have close semantic meaning. There are a variety of algorithms that are used to create clusters of words from text. We chose K-means clustering, which generates a set of $\mathrm{k}$ cluster centers and assigns each word vector to one of the centers. The algorithm is based on minimizing the variance of the Euclidean distance of the points to their nearest cluster centers [10]. We chose K-means clustering because of its simplicity in both implementation and run-time. In this context, each of the clusters are considered a topic found within the text. We discerned these topics by qualitatively analyzing the most commonly used words found in each cluster.

To evaluate the occurrence of topic clusters in the corpus we first consider matrices that map each post/response to their words, as seen in figure 1 and 2 .

$$
M \text { Posts }\left[\begin{array}{cccc}
a_{11} & a_{12} & \cdots & a_{1 N} \\
a_{21} & a_{22} & \cdots & a_{2 N} \\
\vdots & \vdots & \ddots & \vdots \\
a_{M 1} & a_{M 2} & \cdots & a_{M N}
\end{array}\right]
$$

Figure 1: Posts by Words Matrix $(P W)$

$$
M * \text { Responses }\left[\begin{array}{cccc}
b_{11} & b_{12} & \cdots & b_{1 N} \\
b_{21} & b_{22} & \cdots & b_{2 N} \\
\vdots & \vdots & \ddots & \vdots \\
b_{M 1} & b_{M 2} & \cdots & b_{M N}
\end{array}\right]
$$

Figure 2: Responses by Words Matrix $(R W)$

Both matrices map the posts/responses to their frequency of each word in the corpus. The $M^{*}$ responses denotes that each row represents all responses to one post, so that there are the same number of response rows as posts. The cell $\mathrm{a}_{11}$ denotes the frequency of the first word in the first row post/response. Since each matrix is the same size and ordered by post, we then concatenated the two matrices in order to align all response text to the parent post, shown in figure 3.

$\mathrm{M}^{*}$ Posts/Responses $\left[\begin{array}{cccccccc}a_{11} & a_{12} & \cdots & a_{1 N} & b_{11} & b_{12} & \cdots & b_{1 N} \\ & & & & & & & \\ a_{21} & a_{22} & \cdots & a_{2 N} & b_{21} & b_{22} & \cdots & b_{2 N} \\ \vdots & \vdots & \ddots & \vdots & \vdots & \vdots & \ddots & \vdots \\ a_{M 1} & a_{M 2} & \cdots & a_{M N} & b_{M 1} & b_{M 2} & \cdots & b_{M N}\end{array}\right]$

Figure 3: Posts and Responses By Words matrix $(P R W)$ produced through matrix concatenation

Next, after performing K-means clustering, we produced a binary matrix that shows which cluster each word in the corpus belongs to, as seen in figure 4 .

$$
N \text { Words }\left[\begin{array}{cccc}
c_{11} & c_{12} & \cdots & c_{1 L} \\
c_{21} & c_{22} & \cdots & c_{2 L} \\
\vdots & \vdots & \ddots & \vdots \\
c_{N 1} & c_{N 2} & \cdots & c_{N L}
\end{array}\right]
$$

Figure 4: Words By Clusters Matrix (WC)

By using matrix multiplication, we can then obtain a matrix that shows the clusters that appear in each post and response. Each cell in the matrix represents how many occurrences there are of a specific cluster within a post or response.

$$
\mathrm{M}^{*} \text { Posts/Responses }\left[\begin{array}{cccccccc}
c_{\mathrm{P} 11} & c_{\mathrm{P} 12} & \cdots & c_{\mathrm{P} 1 L} & c_{\mathrm{R} 11} & c_{\mathrm{R} 12} & \cdots & c_{\mathrm{R} 1 L} \\
& & & & & & & \\
c_{\mathrm{P} 21} & c_{\mathrm{P} 22} & \cdots & c_{\mathrm{P} 1 L} & c_{\mathrm{R} 21} & c_{\mathrm{R} 22} & \cdots & c_{\mathrm{R} 2 L} \\
\vdots & \vdots & \ddots & \vdots & \vdots & \vdots & \ddots & \vdots \\
c_{\mathrm{P} M 1} & c_{\mathrm{P} M 2} & \cdots & c_{\mathrm{P} M L} & c_{\mathrm{P} M 1} & c_{\mathrm{P} M 2} & \cdots & c_{\mathrm{P} M L}
\end{array}\right]
$$

Figure 5: Posts and Responses By Clusters matrix $(P R C)$ produced through matrix multiplication

Once we obtained this matrix, we normalized the values by summing the entirety of the rows and normalizing the resultant vector to get a vector of relative cluster frequency within each post and response. L1 normalization was used for this purpose.

L1 normalization was performed both before and after the summation of each row in the Post/Response Cluster matrix in order to increase accuracy. Normalizing prior to summation ensured that both the posts and responses were weighed equally when calculating the overall cluster frequency within the two categories. This is important because if a single post or response has an abnormally large amount of occurrences of a single cluster, this could bias the 
cluster frequency towards its respective category. Normalizing after row summation allowed for us to represent each cluster frequency as a percentage between 0 and 1 , which simplifies analysis and creates a scale on which we can compare the relative cluster frequency between posts and comments.

\subsection{Association Rules}

Our final analysis step was the mining of association rules from our data using the frequent pattern growth algorithm. This algorithm works by generating frequent itemsets using a FP-tree [28]. In order to obtain meaningful association rules, we generated new clusters from two models: one trained on all text from the posts, and one trained on all text from the responses. Therefore, our Post-Response to Cluster matrix was generated using two sets of clusters obtained from each respective category, as seen in figure 6.

$$
\mathrm{M}^{*} \text { Posts/Responses }\left[\begin{array}{cccccccc}
c_{11} & c_{12} & \cdots & c_{1 L} & k_{11} & k_{12} & \cdots & k_{1 L} \\
& & & & & & & \\
c_{21} & c_{22} & \cdots & c_{2 L} & k_{21} & k_{22} & \cdots & k_{2 L} \\
\vdots & \vdots & \ddots & \vdots & \vdots & \vdots & \ddots & \vdots \\
c_{M 1} & c_{M 2} & \cdots & c_{M L} & k_{M 1} & k_{M 2} & \cdots & k_{M L}
\end{array}\right]
$$

\section{Figure 6: Posts and Responses by Clusters Matrix $(P R C K)$}

We analyzed all one-to-one association rules and focused on the rules with post clusters on the left-hand side and response clusters on the right-hand side in order to see correlation between a cluster that appears in a post to a cluster that appears in any of the direct responses to the post.

\section{R/SUICIDEWATCH}

Data was collected from Reddit, a social network built off a massive collection of forums in which users can post and respond to content. According to Alexa, Reddit is the 6th most visited website in the U.S [2]. Reddit is organized into subreddits, which are forums defined by specific topics or rules regarding what content can be posted. These topics range from news and politics to forums discussing personal relationships, financial problems, or illness.

$\mathrm{r} /$ SuicideWatch is a subreddit for suicidal users to express themselves and receive support. In a typical post, a user may discuss suicidal ideations, depressive symptoms, and other topics related to suicide. A typical response may include personal words of comfort, resources, similar experiences, or suggestions for coping methods. According to the site, SuicideWatch began in 2008 and has grown from a couple thousand members to over 148,000 members.

We chose this subreddit based on a few key factors. The dataset obtained from SuicideWatch is large given the amount of time that it has existed as well as the amount of members. Posts are text-based and focused on personal accounts of suicidal ideation, therefore the data is clean and relevant to our topic of interest. It also has volunteer users, known as moderators, who remove posts and responses that do not follow the community guidelines of the subreddit. Moreover, the serious nature of the forum greatly reduces the risk of our data containing inappropriate content.

After collecting the data, we removed empty posts in which the content was deleted, either by the user or moderators. We then removed hyperlinks and concatenated the post title to the rest of the text to make the data more uniform. Finally, we removed punctuation, capitalization, and any special characters in order to focus on the words themselves.

After cleaning the data, we obtained 228,962 posts and 376,604 responses. We also obtained a subset of posts with direct responses within the dataset by matching comments and posts with the same link id. Of the 228,962 posts, 56,469 had archived direct responses.

\section{RESPONDING TO SUICIDAL IDEATION}

Researchers previously leverage $r$ /SuicideWatch to evaluate the risk factors for suicide. Risk factors are characteristics of a person or their environment which increase the likelihood of suicide [1]. In this work, we also see such risk factors within our clustering results. Some of the major risk factors that we detected in this work include mental disorders, feelings of hopelessness, personal or financial loss, and a history of abuse. In this effort, we report protective factors, personal or environmental characteristics which lower an individual's risk of suicide. Such characteristics have not been as rigorously or extensively researched as risk factors [6]. Policy and practice around suicide intervention requires accurate knowledge about the conditions and factors that both increase and decrease an individual's risk of suicide [24].

According to Mental Health America, 8 out of 10 people considering suicide give some sign of their intentions. Furthermore, 64 percent of those who attempt suicide seek medical care in the month leading up to the attempt [5]. Properly identifying suicidal ideation and responding appropriately can have a huge impact on the outcome for suicidal individuals.

There are a variety of online resources that identify effective methods for responding to suicidal ideation. Due to limited research on the efficacy of suicide prevention methods, response approaches often focus on the encouragement of protective factors such as clinical care, a sense of connectedness, and development of coping skills. Furthermore, the nature of responses should be hopeful and non-judgmental.

Encouraging Clinical Care. The first category of responses surrounds encouraging a suicidal individual to get treatment. This includes suggesting contact with a mental health professional or providing resources such as crisis lines. This category is emphasized for responding to high-risk individuals who have a specified plan for suicide [7]. These types of responses not only provide useful information, but indicate the availability of treatment and the possibility of recovery [8]. Furthermore, a strong and ongoing relationship with a mental health professional is one of the factors that decreases the risk of suicide [1].

Proactivity in Communication. The second category is proactivity in communicating with a suicidal individual. A 2005 study of 200 patients at a public hospital analyzed the risk and protective factors associated with suicide attempts, and found that higher levels of social support predicted a lower rate of attempts [15]. A response that offers a sense of social support is one that is proactive in both listening to and conversing with the suicidal individual. This includes making oneself available and being pointed and specific with methods of contact [7]. Research shows that there is no evidence that asking about suicide increases the likelihood of a 


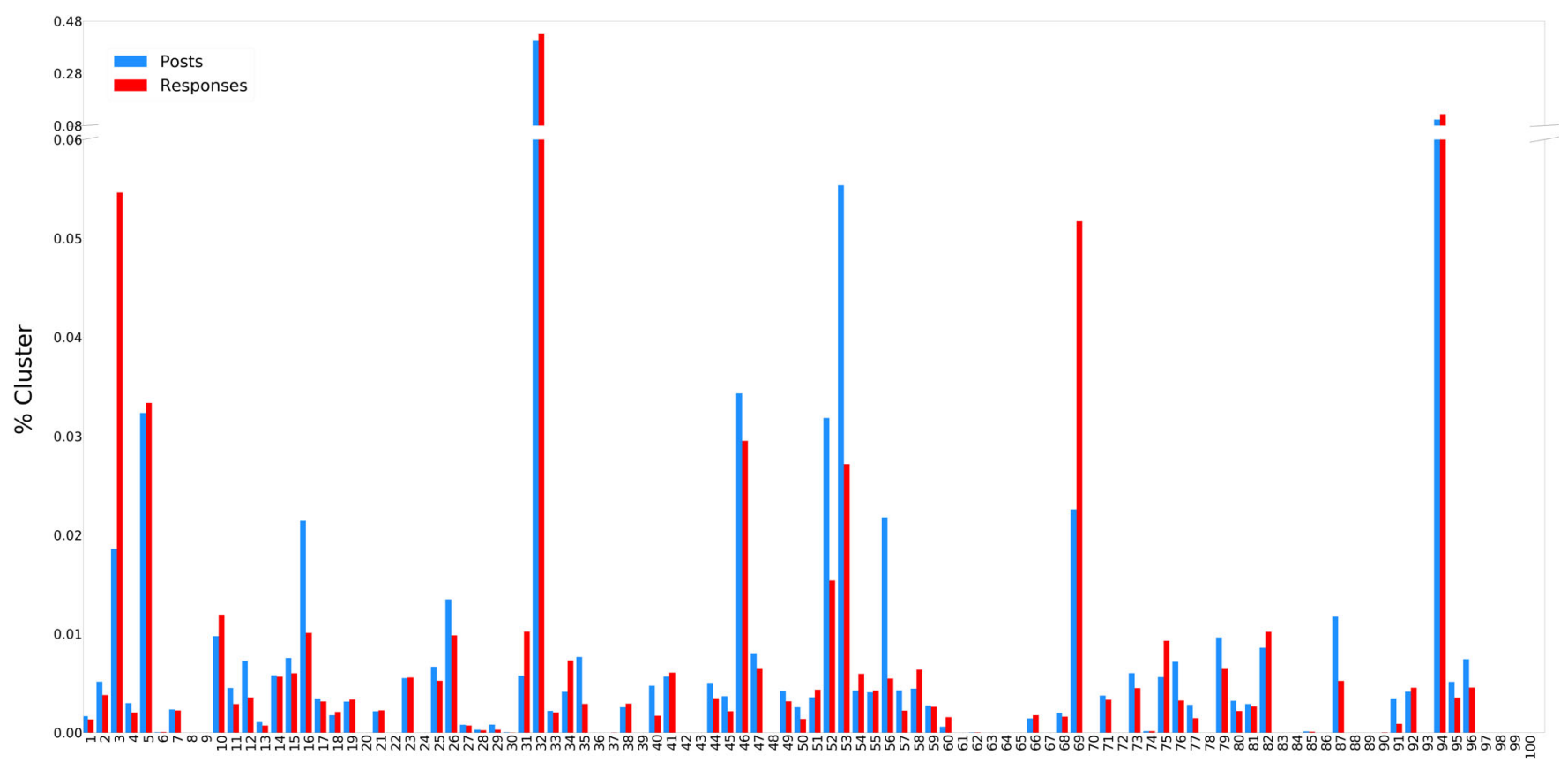

Figure 7: Cluster Frequency In Posts and Responses

This graph depicts the relative frequency of each of the 100 topic clusters generated. The posts and the responses are graphed together for comparison of relative cluster frequency between the two categories. Each number on the x-axis corresponds to a cluster number containing words with similar semantic meaning, and the number on the y-axis corresponds to the relative amount of usage the respective cluster gets in comparison to all other clusters in that category.

person engaging in suicidal behavior [9]. Responses that are direct and proactive create an opportunity of conversation that may have otherwise felt unavailable to the suicidal individual.

Encouragement of Positive Lifestyle Changes. The third category is encouraging positive lifestyle changes and the development of coping mechanisms. This can include advice on improving diet, exercise, and sleep [7]. Guidelines for safe online discussion of suicide also indicate that responses should include information on protective factors like engaging in meaningful activities, such as sports, artistic hobbies, or social groups [8]. A 2003 study which analyzed activities in relation to self-reported suicide attempts among adolescents found that the attitude towards sport as a healthy activity was associated with lower likelihood of suicidal behaviour; and non-attempters turned to sport as a coping behaviour in distress more frequently than attempters [23]. Such activities can provide an individual with a feeling of purpose, an outlet for coping, and a social group. Furthermore, getting enough sleep, physical activity and adequate nutrition decrease depressive symptoms [6].

Sympathetic Sentiment. Lastly, responses to those suffering suicidal ideation should be non-judgmental, sympathetic, and hopeful. Avoiding judgment or strong emotional reactions allows for an individual expressing suicidal ideation to feel connected and validated [7]. Furthermore, responses should include messages of hope. As suicide is associated with feelings of hopelessness, outside reassurance and messages of hope are considered crucial [4]. A 2013 study of patients with depression found that higher scores of optimism and future orientation, or a positive outlook of the future and presence of reasons for living, were associated with lower levels of suicide ideation and lower likelihood of suicide attempts in patients being treated for depression [12]. Hopeful and sympathetic language can increase feelings of optimism and motivation.

\section{EXPERIMENTAL RESULTS}

In this section, we discuss the results obtained from building Word2Vec models off our cleaned data, creating clusters of similar words in the model's vocabulary, and generating association rules between clusters found in the posts and their responses. We focus on the relative cluster frequency between posts and responses and one-to-one associations between post clusters and response clusters.

\subsection{Experimental Details}

The parameters for our Word2Vec model included a 300 dimension vector space, a context window size of five, and a negative sampling rate of five. These parameters were chosen based on results from Mikolov et. al as well as prior work on the same corpus. We trained a Word2Vec model on the entirety of the text from both the posts and responses, as well as two separate models on each category.

For k-means clustering, we tested $k$ at values between 10 and 400 . We visually inspected the sum of squared errors of the generated clusters against $\mathrm{k}$, and found 100 to be at the knee of the graph. This value allowed for a variety of distinct word clusters, while still remaining efficient in the generation of clusters. 
Association rules were analyzed based on three values: support, confidence, and lift. Support indicates the probability that the two clusters within the rule appeared in the same text, which in this case is all text from a post and its responses. Confidence is the probability of both clusters occurring together (support) divided by the probability of the left-hand side cluster occurring. Lift is the probability of both clusters occurring together divided by the probability of the left-hand side cluster occurring and divided by the probability of the right hand side cluster occurring. Itemsets, or groupings of clusters that appear together, were mined at a support level of 7.5 percent in order to gather as many itemsets as possible within a reasonable run-time. We set a threshold confidence level of 10 percent for the association rules generated from these itemsets. We kept our support and confidence levels relatively low in order to maximize our set of possibly meaningful association rules.

\subsection{Comparative Cluster Frequency}

The graph shown in figure 7 depicts the relative usage of each cluster within the posts, shown in blue, as compared to the responses shown in red. The horizontal axis represents the cluster number and the vertical axis represents the relative frequency of the cluster as a percentage. The percentage of use is calculated with respect to every other cluster for that category. This graph allows us to visualize how often topics are discussed in posts as compared to in the responses. The peaks at cluster 32 and cluster 93 are noticeably high for both categories. This is to be expected as both clusters contain very general language. For example, cluster 32 contains the words "i'm", "like", and "feel", and cluster 93 contains words such as "get", "things", and "go".

While these clusters were most popular, this does not indicate that they are most important, as neither cluster represents a a focused topic within the language. Rather, this language is more indicative of words that are commonly used in the English language. We found that meaningful analysis came from investigation of clusters that had a stark difference between the two peaks. For example, cluster 53 is about twice as popular for posts as it is for comments. This cluster contains words surrounding death and suicide, including "nothing", "end", "kill", "die", and "suicide". This words are more common in the originating posts, often pleas for help, than in the responses, often expressions of support.

Cluster 69 was much more prominent for responses, and includes words such as "talk", "listen", "wanna", "hear", "pm", and "tonight". In this context, "pm" is a common abbreviation for private messaging. From these words we can discern that this cluster surrounds the initiation or offer of conversation between two users. This strong favorance towards responses indicates that it is much more common for users responding to a post to offer to listen or have a one-onone conversation. This example indicates that responders within the subreddit are implementing protective methods whether intentional or not [6]. Recall that the CDC defines one of the protective factors for suicide as community support or connectedness.

A few groupings of cluster preferences pointed towards common themes. For example, clusters 46, 56, and 76 each have a higher favorance for posts than comments. Cluster 46 includes profanity as well as "hate" and "stupid". These words portray anger or frustration. Meanwhile, cluster 56 contains words such as "lonely", "miserable", "broken", and "hopeless", which indicate depressive feelings. Finally, cluster 76 contains words such as "worthless", "useless", "ugly", and "pathetic". These words appear to express feelings of isolation, hopelessness, misery, and worthlessness, which are considered risk factors for suicidal ideation and common depressive symptoms. The clusters that have extremely negative or pessimistic subjective language appear to be strongly associated with posts. In contrast, responses favor more positive language such as cluster 82, which includes "dream", "successful", "value", and "potential".

Certain themes emerged among clusters that were favored by the responses. The peaks at clusters 31,41 , and 60 each show a sizeable trend towards responses. Cluster 31 contains words such as "call", "therapist", "phone", and "professional", indicating that this cluster surrounds the topic of seeking professional medical help. Cluster 41 also references professional treatment for mental health, including words such as "therapy", "medication", "psychiatrist", and "treatment". Lastly, cluster 60 contains the trigram "suggest seek help", the bigram "professional help", and the words "psychologist" and "recommend". This cluster again surrounds the topic of professional help with the added personal layer of words like "recommend" and "suggest". These trends suggest that language surrounding professional help is more likely to be a response or recommendation within a comment than an element mentioned in the original post. In addition, these responses again reflect strong protective measures as clinical care is cited as a key protective factor against suicide [6].

Clusters surrounding school and work also show an interesting contrast between the language of posts and the language of responses. Cluster 11 is favored by posts, and includes words and phrases like "high school", "grades", "failed", and "drop". Meanwhile, cluster 58 is favored by responses and includes "read", "writing", "interesting", "history", and "subject". It appears that while both categories discuss the topic of school, posts favor language surrounding performance and grades, while responses favor language surrounding content and interests. In fact, the only cluster directly discussing interests is cluster 34 and is highly preferred by comments. This cluster contains words such as "fun", "music", "hobbies", and "interests". This could indicate that responses suggest finding activities or subjects that interest the sufferer. A common risk factor for suicidal ideation is loss of interest or motivation for activities such as hobbies or school, which may provide some reasoning for the lower usage of these cluster topics among posts [6].

Many clusters with higher frequency in the originating posts are indicative of events or circumstances that are defined as risk factors for suicide. Both Cluster 2, which discusses debt with words and phrases such as "money", "debt", and "homeless" and Cluster 71, which describes job loss, with words and phrases such as "job", "company", "fired", and "quit job" were favored by posts. The CDC defines loss as a risk factor, including relational, work, or financial loss [3]. Clusters describing mental illness were also favored by the posts, such as cluster 40 and cluster 73 . Cluster 40 focuses on substance abuse, with words such as "pills", "drank", "heroin", and "od" and cluster 73 discusses mental disorders, including words such as "depression", "anxiety", "bipolar", and "ptsd". The CDC cites a history of substance abuse and mental disorders as two predominant risk factors for suicide [3]. Risk factors also include a history of abuse, which appears to be the topic of cluster 50 with words like 


\begin{tabular}{|l|l|l|l|l|l|}
\hline Cluster 32 & Cluster 45 & Cluster 67 & Cluster 35 & Cluster 36 & Cluster 68 \\
\hline suicide & stupid & failed & friends & life & deserve \\
\hline thinking & selfish & class & outside & nothing & failure \\
\hline thoughts & crazy & fail & meet & future & worthless \\
\hline mind & attention & grades & social & worth & miserable \\
\hline killing & lazy & classes & relationships & continue & burden \\
\hline often & sound & failing & group & motivation & useless \\
\hline ending & ass & study & comfortable & purpose & pathetic \\
\hline lately & dumb & drop & isolated & whats_point & human \\
\hline ways & asshole & semester & awkward & dreams & weak \\
\hline daily & bitch & studying & rarely & effort & good_enough \\
\hline Cluster 156 & Cluster 177 & Cluster 196 & Cluster 139 & Cluster 144 & Cluster 162 \\
\hline contact & help & want & walk & things & im \\
\hline resources & getting & talk & bed & better & sorry \\
\hline available & talking & need & spend & change & hey \\
\hline line & therapist & someone & exercise & possible & sounds_like \\
\hline mental_health & doctor & id & run & ways & relate \\
\hline information & therapy & pm & eat & control & sounds \\
\hline provide & perhaps & listen & room & small & hi \\
\hline medical & anxiety & hear & food & positive & sorry_hear \\
\hline local & professional & free & wake & perspective & hey_man \\
\hline calling & helpful & youd & relax & lots & rough \\
\hline
\end{tabular}

Figure 8: Table of Relevant Topic Clusters by Top 10 Words

Top Words were sorted by total frequency of appearance across all posts and responses. The clusters in this figure represent some of the most common clusters involved in the motifs found in our association rules.

"childhood", "abused", "raped", and "bullying", and physical illness, which appears as the topic of cluster 68 with words and phrases like "cancer", "disease", "chronic", and "heart attack". Although online discussion of suicide is informal compared to clinical settings, our analysis suggests that the informal topics found within these forums agree with a large number of previously defined risk factors.

\subsection{Association Rules}

Association rules were mined at a support level of 7.5 percent and a confidence level of 10 percent. We focused on rules with above-average lifts as this value is most indicative of the correlation between clusters within the rule. In order to provide a thorough analysis of the rules, we searched for coherent motifs that captured overarching trends. We focused on rules containing post clusters that related to the risk factors for suicide, response clusters that related to preventative factors, and clusters with a clear positive or negative sentiment. Within this subset, we focused on rules with lifts that were higher than the average lift for all rules containing those clusters.

Encouraging Clinical Care. A key motif that we identified within the association rules was that responders often try to direct the poster to mental health professionals. For example, the rules Cluster 32 -> Cluster 156 and Cluster 32 -> Cluster 177 shows that when words from Cluster 32, which represents suicidal ideation, are present in an originating post, words from clusters 156 and 177 often show up in the response. These clusters represent mental health resources and professionals. The common words of the clusters can be seen in Figure 9. The encouragement of care such as crisis resources or contact with a mental health professional are highly recommended by the CDC when conversing with a suicidal individual, particularly those who appear to be at risk of attempting in the near future.

Proactivity in Communication. Another motif that we identified was the notion of proactivity in establishing contact within responses. This pattern was evident in the rules Cluster 45 -> Cluster 196, Cluster 67 -> Cluster 196, Cluster 35 -> Cluster 196, When posters express self loathing language, concern about school, or trouble fitting in, responders are more often to reply with words such as "talk", "pm" or "listen". Again, the responses appear to be falling within the suggested guideline of being proactive and personal. As well, personal responses seem to occur more frequently with posts that discuss negative relational experiences.

Encouragement of Positive Lifestyle Changes. We also identified the encouragement of positive lifestyle changes as another motif within our association rules. Cluster 36 -> 139, Cluster 36 -> 187 are rules that show this pattern. The words in Cluster 36 seem to represent hopelessness and a sense of dread about the future. In response to those ideas, comments suggest "walk", "exercise", and "food" found in cluster 139 or "music", "hobbies" and "book" found in cluster 187. Similar responses were observed when users expressed negative feelings such as "sad", "lonely", "angry", "anxious", and "stressed". These words often evoked the same encouragement to adopt healthy lifestyle choices.

Sympathetic Sentiment. Another common motif demonstrated sympathetic and hopeful sentiment within responses. The rules Cluster 45 -> Cluster 144 and Cluster 45 -> 162 show that "stupid", 
"selfish" and "crazy" are often met with "better", "change", and "possible" or "sorry", "relate" and "sounds". When poster express self loathing, responders are more likely to express optimism about the future or simply express empathy. Likewise, "failure", "worthless" and "miserable" evoked similar responses. Again, it appears that responders in $\mathrm{r} /$ SuicideWatch are practicing many of the guidelines described by mental health care professionals.

$\mathrm{r} /$ SuicideWatch is a rich and diverse community. We chose the above motifs because they reflect the ways in which responses within the subreddit are implementing protective factors. The automated detection of protective factors within our corpus of responses allows us to report that the support offered in SuicideWatch aligns with expertly-defined methods of support for suicidal individuals. This analysis can set the groundwork for future studies on the efficacy of online forums for suicidal individuals, as well as the efficacy of these methods of responding.

\section{CONCLUSION}

In this work, we automatically extracted informal topics from social media posts expressing suicidal ideations and their responses. We first compared the relative frequency of latent topics between the posts and the comments. In general, we found that our informal topics that represent suicidal risk factors occurred with high frequency within the posts, while clusters that discuss protective factors such as treatment and coping skills occurred with high frequency within the responses. Furthermore, we found that clusters with similar topics but different sentiment differed in frequency, with posts favoring negative sentiment and responses favoring positive sentiment. We also analyzed association rules generated from posts and their respective responses. We found that the rules contained response clusters that represent different categorical, expert-defined methods of responding to suicidal ideation. Furthermore, clusters that represented different risk factors within the posts had associations to different response methods. In short, our analysis of posts discussing suicidal ideation and their responses suggests that user responses are primarily positive in sentiment and adequately implement expertly-defined methods of responding to suicidal ideation. These findings allow us to better understand the efficacy of such mental health forums for suicidal individuals. Future work could use these analysis techniques to track a specific user's discussion topics over time in order to identify factors that contribute to the evolution of their mental illness.

\section{REFERENCES}

[1] [n. d.]. Risk and Protective Factors. https://www.sprc.org/about-suicide/riskprotective-factors

[2] [n. d.]. Top Sites in United States. https://www.alexa.com/topsites/countries/US

[3] 2017. 10 Leading Causes of Death, United States. Technical Report. Centers for Disease Control and Prevention. https://webappa.cdc.gov/cgi-bin/broker.exe

[4] 2018. How to Help Someone Who is Suicidal. https://www.mentalhealthfirstaid org/2018/12/how-to-help-someone-who-is-suicidal/

[5] 2018. Suicide. https://www.mentalhealthamerica.net/suicide\#ref2

[6] 2018. Suicide Risk and Protective Factors. https://www.cdc.gov/ violenceprevention/suicide/riskprotectivefactors.html

[7] 2019. Suicide Prevention. https://www.helpguide.org/articles/suicideprevention/suicide-prevention.htm

[8] 2019. Tips for discussing suicide on social media - A guide for youth. https://neuroscience.stanford.edu/news/tips-discussing-suicide-socialmedia-guide-youth
[9] Caroline A Blades, Werner GK Stritzke, Andrew C Page, and Julia D Brown. 2018. The benefits and risks of asking research participants about suicide: A metaanalysis of the impact of exposure to suicide-related content. Clinical psychology review 64 (2018), 1-12.

[10] Johannes Blömer, Christiane Lammersen, Melanie Schmidt, and Christian Sohler. 2016. Theoretical analysis of the k-means algorithm-a survey. In Algorithm Engineering. Springer, 81-116.

[11] Rafael A. Calvo, David N. Milne, M. Sazzad Hussain, and Helen Christensen. 2017. Natural language processing in mental health applications using nonclinical texts. Natural Language Engineering 23, 5 (2017), 649âĂŞ685. https: //doi.org/10.1017/S1351324916000383

[12] Edward C Chang, A Yu Elizabeth, Jenny Y Lee, Jameson K Hirsch, Yvonne Kupfermann, and Emma R Kahle. 2013. An examination of optimism/pessimism and suicide risk in primary care patients: does belief in a changeable future make a difference? Cognitive Therapy and Research 37, 4 (2013), 796-804.

[13] Gobinda G Chowdhury. 2003. Natural language processing. Annual review of information science and technology 37, 1 (2003), 51-89.

[14] J Clement. 2019. U.S. Reddit reach by age group 2019. https://www.statista.com/ statistics/261766/share-of-us-internet-users-who-use-reddit-by-age-group/

[15] Michael T Compton, Nancy J Thompson, and Nadine J Kaslow. 2005. Social environment factors associated with suicide attempt among low-income African Americans: The protective role of family relationships and social support. Social psychiatry and psychiatric epidemiology 40, 3 (2005), 175-185.

[16] Glen Coppersmith, Mark Dredze, Craig Harman, and Kristy Hollingshead. 2015. From ADHD to SAD: Analyzing the language of mental health on Twitter through self-reported diagnoses. In Proceedings of the 2nd Workshop on Computational Linguistics and Clinical Psychology: From Linguistic Signal to Clinical Reality. $1-10$.

[17] Munmun De Choudhury, Emre Kiciman, Mark Dredze, Glen Coppersmith, and Mrinal Kumar. 2016. Discovering shifts to suicidal ideation from mental health content in social media. In Proceedings of the 2016 CHI conference on human factors in computing systems. ACM, 2098-2110.

[18] Björn Gambäck and Utpal Kumar Sikdar. 2017. Using Convolutional Neural Networks to Classify Hate-Speech. In Proceedings of the First Workshop on Abusive Language Online. Association for Computational Linguistics, 85-90. https: //www.aclweb.org/anthology/W17-3013

[19] Reilly N Grant, David Kucher, Ana M León, Jonathan F Gemmell, Daniela S Raicu, and Samah J Fodeh. 2018. Automatic extraction of informal topics from online suicidal ideation. BMC bioinformatics 19, 8 (2018), 211.

[20] Christopher Homan, Ravdeep Johar, Tong Liu, Megan Lytle, Vincent Silenzio, and Cecilia Ovesdotter Alm. 2014. Toward macro-insights for suicide prevention: Analyzing fine-grained distress at scale. In Proceedings of the Workshop on Computational Linguistics and Clinical Psychology: From Linguistic Signal to Clinical Reality. 107-117.

[21] Hootsuite Media Inc. [n. d.]. Digital in 2019 - Social Media Marketing Management Dashboard. https://hootsuite.com/pages/digital-in-2019

[22] Siwei Lai, Kang Liu, Shizhu He, and Jun Zhao. 2016. How to generate a good word embedding. IEEE Intelligent Systems 31, 6 (2016), 5-14.

[23] Andrej Marušič, Sabine Landau, and Martina Tomori. 2003. Long-term Trends, Seasonality, Weekly Distribution, and Methodsof Suicide in Slovenia: A Comparison Between the Younger and Older Population. Archives of suicide research 7, 2 (2003), 135-143.

[24] J McLean, M Maxwell, S Platt, F Harris, and R Jepson. 2008. A systematic international literature review of review-level data on suicide risk factors and primary evidence of protective factors against suicide. Edinburgh: Scottish Government (2008).

[25] Tomas Mikolov, Kai Chen, Greg Corrado, and Jeffrey Dean. 2013. Efficient estimation of word representations in vector space. arXiv preprint arXiv:1301.3781 (2013).

[26] Tomas Mikolov, Ilya Sutskever, Kai Chen, Greg S Corrado, and Jeff Dean. 2013. Distributed representations of words and phrases and their compositionality. In Advances in neural information processing systems. 3111-3119.

[27] Victoria Nebot and Rafael Berlanga. 2012. Finding association rules in semantic web data. Knowledge-Based Systems 25, 1 (2012), 51 - 62. https://doi.org/10.1016/ j.knosys.2011.05.009 Special Issue on New Trends in Data Mining.

[28] B. Nigam, A. Nigam, and P. Dalal. 2017. Comparative Study of Top 10 Algorithms for Association Rule Mining. International fournal of Computer Sciences and Engineering 5, 8 (Aug 2017), 190âĂŞ195. https://doi.org/10.26438/ijcse/v5i8. 190195

[29] Matthew K Nock, Guilherme Borges, Evelyn J Bromet, Christine B Cha, Ronald C Kessler, and Sing Lee. 2008. Suicide and suicidal behavior. Epidemiologic reviews 30, 1 (2008), 133-154.

[30] Gustavo Turecki and David A Brent. 2016. Suicide and suicidal behaviour. The Lancet 387, 10024 (2016), 1227-1239.

[31] Tom Young, Devamanyu Hazarika, Soujanya Poria, and Erik Cambria. 2018. Recent trends in deep learning based natural language processing. ieee Computational intelligenCe magazine 13, 3 (2018), 55-75. 